\title{
Design of Distributed Control and Emergency Shutdown System for Urea and Ammonia Plant
}

\author{
Radha Ravindra Borikar ${ }^{1}$ and Monica Subashini $\mathrm{M}^{2 *}$ \\ ${ }^{1}$ Department of Control and Automation, School of Electrical Engineering, VIT, India \\ ${ }^{2}$ Department of Control and Automation, School of Electrical Engineering, VIT, India
}

\begin{abstract}
In modern process industries, the need for automation has become necessary to improve the feasibility of production. Automation reduces the possibility of human error and also ensures accuracy of output. In automation and process industries, DCS is used to control several processes being carried out simultaneously with minimum scope for failure. In this project, we have proposed a design on Distributed Control System and Emergency Shutdown System which controls the processing of urea and ammonia. The software used for the Distributed Control System is Centum VP (Vigilant Plant) and the logic designer tool is used to design the control logics of the DCS and ESD.
\end{abstract}

\section{Introduction}

A distributed control system has become more and more popular nowadays in the process industries as it is useful for many complex control processes[1],[2],[3]. There are many vendors who provide Safety solutions and Distributed control systems like Emerson, Honeywell, Yokogawa etc. There are several advantages of DCS over other control systems[9]. It has very flexible architecture hardware as well as the software tool. It has vigorous communication systems among field control stations, field instruments, and operating stations. For the design of process plants in the Chemical and Fertilizer industry sector there are different design constraints[10], safety is the primary design constraint while designing processing plants for the Chemical and Fertilizer industry, hence all the different international safety standards and norms should be satisfied[11].Designing and commissioning of large scale process plants are a reality due to the advancements made in the field of process control and Automation. For the design of process plants in the Chemical and Fertilizer industry sector there are different design constraints, safety is the primary design constraint while designing processing plants for the Chemical and Fertilizer industry, hence all the different international safety standards and norms should be satisfied.

\footnotetext{
* Corresponding author: monicasubashini.m@gmail.com
} 
[8] This reference paper used Object Oriented Analysis and Design software engineering proposal to model Distributed Digital control system of power plant. They used Failure mode and effect analysis (FMEA) process for detailed and systematic examination of the operation. [4]The researchers have implemented the control system for an Acid solution formation plant on APROL DCS System which is Linux based system. On APROL both batch and continuous processes can be executed. For controlling the plant, the logic and algorithms are applied in safe, manual and auto mode. The authors [12] have implemented bottle filling system with the use of Distributed control system for process control management. Here, MATLAB software is used for implementation of bottle filling system which is based on DCS. [5] The authors have used DCS using ultrafiltration ceramic membrane filtration for a sewage treatment which has three layers: Operation station, Field control station (FCS), field instrument layer. Visual basic is the application software of sewage treatment which is adapted by block-based design.

[7]This paper presents OLE for process control (OPC) and DCS to connect simulation system as well as in order to get data shared by various industries. By this, for the training of engineers, DCS commissioning or maintenance, verification test of the control strategy, the connected system can be utilized to avoid exploding equipment for improving improper parameters and decrease the cost of commissioning.

\section{Methodology}

The overall design of the plant is done based on the logics for the functioning of all the different field instruments present such as Transmitters, Actuators, Valves etc., along with the Piping \& Instrumentation Diagrams (P\&ID)available. The total number and type of Input - Output is assessed based on this document. Predefined function blocks are used for compiling the logic programs. The Field Controller Station (FCS) is loaded with these developed function blocks to perform the overall control scheme. The design and implementation of the ESD is based on the Cause \& Effect matrix. All the hardware systems are redundant in nature and this approach of redundancy is to prevent the failure of the complete control system in case of a single module failure. By default the communication protocols and the overall control logics used will be largely proprietary depending on the supplier of the control system.

\section{Hardware design criteria}

\subsection{Distributed control system}

Distributed Control System or DCS is a control system that is designed for a process plant. In DCS, independent controllers are distributed in the system under the supervision of a central supervisory control. The excellent reliability and safety features of the DCS system makes it perfect for implementation in large scale continuous processing plants. 


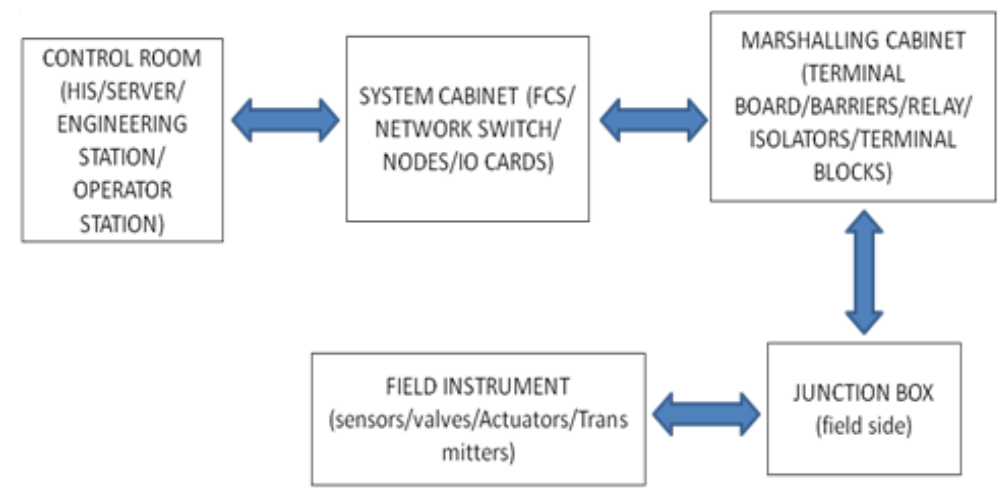

Fig. 1. Block Diagram of Signal flow in Distributed Control System

Figure 1 depicts the signal flow from the Human Interface Station (HIS) to the Field instruments and vice versa. The signal from the HIS or Engineering Station is sent to the System cabinet where the Controller of DCS system called as Field Control Station (FCS) is present. The FCS has the Logic Blocks present in it and does the processing of the signals. Once logic processing is done the signal goes to the IO card modules. The signal is converted to the corresponding type i.e. Analog or Digital as per the respective IO card module; it then travels via Pre-Fab cables to the Marshalling Cabinet. The Pre-Fab cable terminates at the Terminal Boards housed in the marshalling cabinet, the terminal boards can be Analog input/output or Digital input/output as per the signal type.

The Output signal from the DCS is generated at the Human Machine Interface (HIS) by the engineering operator; this goes to the System cabinet which houses the Field control station node, Network switches, the power supply and IO module cards. The FCS is essentially the DCS controller. The signal after processing passes to the Marshalling cabinet which consists of different components such as Terminal boards, intrinsic safety barriers, relays, Terminal blocks, Isolators etc. from here the signal travels to the Junction boxes wherein the signals are paired and sent to the appropriate sections in the field area.

There are a total of 16 cabinets including the system cabinet, PDB cabinet, analog marshalling cabinet and digital marshalling cabinet. The system cabinet houses the controller, Input-Output modules, Ethernet switches, Communication modules. The PDB cabinet houses the different power bus bars which is part of the power distribution hardware. The power distribution of the system is an important design constraint. Power is taken from UPS as well as Non-UPS power supply, the power given to the Fans, Lights, Fan Failure Detection Unit (FFDU) and RCPT in the cabinets is Non-UPS power supply. The power supplied to all the other components in the cabinets will be from the UPS power supply.

All the Hardware components in the system cabinet will be redundant in nature; this is done as a precaution to avoid a complete system failure of the Distributed Control System (DCS). The Analog signals are in the range of 4-20 mA; this is done for Linearity of the detection and for easier fault detection. The Digital signals are $0 \& 1$.

\subsection{Emergency shutdown system}

Emergency shutdown system (SIS) is dependent system takes the output to predetermined safe state in case of process or system abnormality. It is used in the project to reduce the risk associated with the process. The main control action is performed by the Distributed Control System (DCS); in case of any emergencies during the process the Emergency Shutdown 
(ESD) system comes into action and shuts down the plant in a controlled manner. The software tool used for this is ProSafe-RS.

The safety control station (SCS) is connected to a Vnet/IP domain and communicates with other stations. The SCS consists of the safety control unit (SCU), safety node units (SNUs), unit for optical ESB repeater module, and N-IO nodes. The SCU, SNU, and unit for optical ESB repeater module are collectively called FIO nodes.An analog input module receives analog signals and converts them into internal data of SCU. An analog output module converts the internal data of SCU into analog signals and outputs them. A digital input module receives digital (discrete) signals and converts them into internal data of SCU. A digital output module converts the internal data of SCU into digital (discrete) signals and outputs them.

\section{Software design criteria}

The software used here is CENTUM VP-R6 is essentially used for developing the functional loops and the graphics of the various processes of the plant. The graphics are developed from the functional loops which are in turn designed with reference to the P\&IDs received from the customer. There are different functions blocks we used to create control loops i.e. open loops (PVI) shown in Figure 2, closed loops (PID), Split loops, cascade loops, ratio control loop.

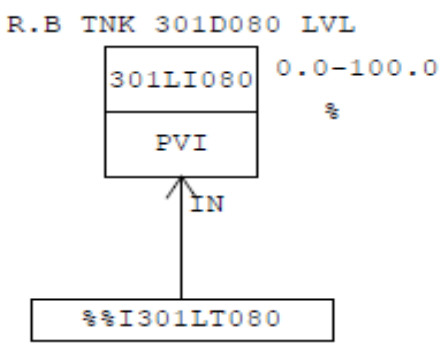

Fig.2. Sample Open Loop Logic Block

The PID Controller Block (PID) [6] provides the most general control function to perform proportional-integral-derivative control based on the deviation of the process variable (PV) from the set point value (SV).Cascade Control has a multiple loop structure, where the output of the controller in the outer loop (primary or master) is the set point of a controller in the inner loop (secondary or slave). The slave measurement is an intermediate process variable that can be used to achieve more effective control of the primary process variable. In the cascade configuration, the process is divided into two parts and therefore two controllers are used but only one process variable is manipulated.

The motor control blocks are provided with function to operate motor -driven pumps and motor-operated valves. The motor control blocks enable starting and stopping of motors from operation and monitoring functions. The Graphic Builder is used to create and edit graphic windows for operation and monitoring. The graphics for the plant depicting the different processes are generated from the P\&IDs. Interlock graphics is generally used to check if that particular valve of that particular process is working properly or not. Figure. 3 shows the example of the interlock graphics. 


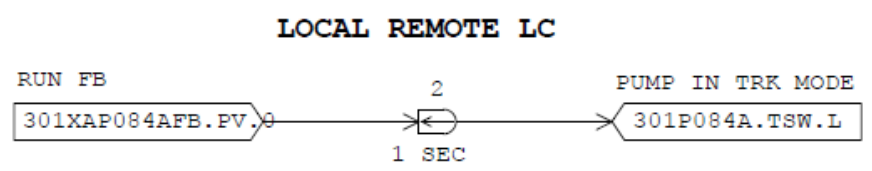

INTERLOCK: 301-IZ-080-RETURN BRINE TANK LEVEL CONTROL

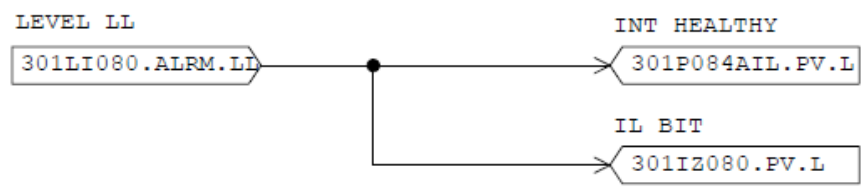

Fig.3. Sample Interlock Logic Function Block

Interlocks in CENTUM VP can be written using either the Sequence Table or the Logic Chart. In a Logic Chart the logic is represented using logic gates in graphical form. Logic Chart consists of input element, output element and logic elements. The total number of inputs, outputs and logic elements that can be configured in one logic chart is 64 . The inputs, logic elements and the outputs are connected by wiring.

\section{Piping and instrumentation diagram (P\&ID)}

Based on the Process Flow Diagram (PFD), the Piping and Instrument Diagram (P\&ID) represents the technical realization of a process with the help of graphical symbols for piping and equipment as well as for control functions and process measurement. Piping and Instrumentation diagram is given by the customers. With the help of this we can differentiate between ESD and DCS tags. Different kind of loops like open loop closed loop can be identified using this. Show all piping connecting equipment, show all valves, and show all instrumentation (measuring, transmitters, controllers, actuators).

\subsection{Graphic View}

A graphic window consists of a schematic representation of a process section showing details of the equipment, process parameters, relevant control loops and process status. Dynamic data is displayed for process variables, alarms and equipment status. All vessel /equipment will be shown in 2D and is depicted in Figure.4. 


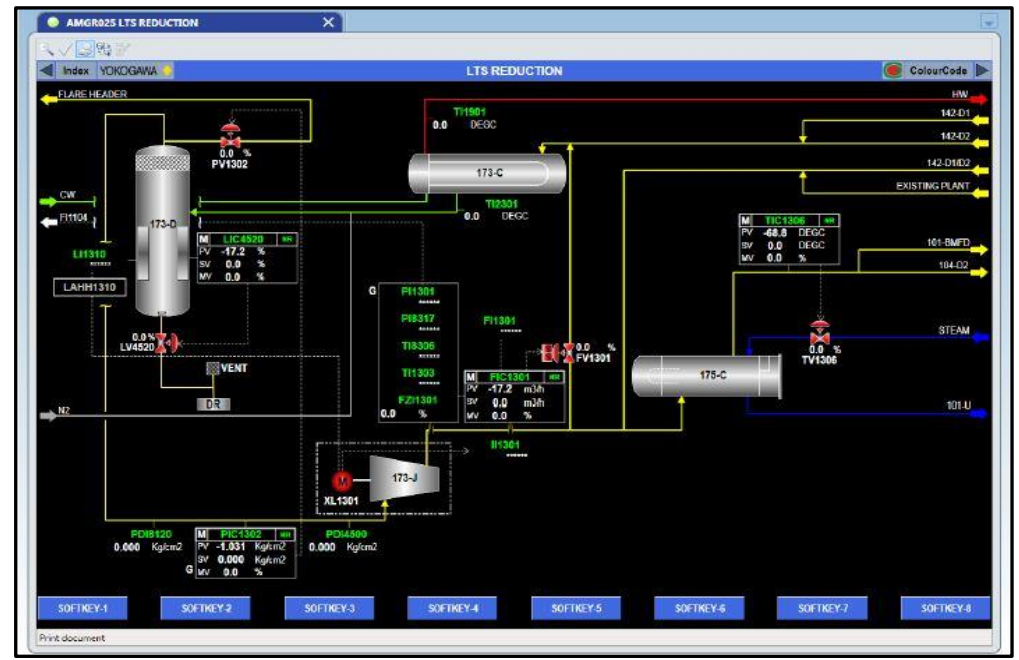

Fig.4. Graphics of LTS Reduction

\section{Results and discussion}

Once the hardware and software part of the project is completed there are mainly 2 levels of testing that must be done to ensure the performance of the system.

- Factory Acceptance Tests

- Site Acceptance Tests

The factory testing is done by the project engineer in presence of the client, whereas the site testing is done by the Field engineering services at the client's factory/plant after connecting the system to all the field instruments present at location. During the Factory Acceptance Testing, complete loop checking of all the hardware and software is carried out using a simulation device for simulating analog and digital signals for the system. Along with which all system checks are carried out including for the redundant parts. Only after the successful completion of the Factory Acceptance Tests the system will be dispatched to the plant for installation.

In general Distributed Control Systems are very expensive, a typical project containing both hardware and software can cost from Rs.1 Crore to Rs.40 Crores and above depending on, the size of the project and its nature of implementation as mentioned in Scope of work document, total number of controllers required, total number of input-output cards and boards, the scope of power distribution, the control scheme \& logics to be implemented and provision of providing Fire \& Gas system (FGS) and Emergency Shutdown System (ESD). The main concern is always the hardware compatibility, since in most plants/process industries/refineries etc. the hardware used will be from different manufacturing companies. This is especially true in the case of up-gradation projects wherein a small section of a functioning plant/industry/refinery is to be upgraded to a more advanced control system.

\section{Conclusion}

Keeping all the above mentioned criteria and also after extensive study of the Scope of work and other plant layout drawings, Piping \& Instrumentation drawing (P\&ID) and discussions with consultants (if required) a detailed project plan is conceived and followed. A general 
design approach is followed in case of projects wherein only the DCS is to be designed and supplied. If there are any special requirements to be incorporated into the control system, such requirements are taken into consideration after detailed site survey and understanding of any existing hardware in the field that has to be integrated into the Distributed Control System. To ensure that there are no compatibility issues, international standards are followed during the design phase of all projects. Hence, future up gradation and revamp of the system can be done without much hassle. As far as the software part is concerned, modifications are easier to accommodate.

\section{References}

1. J. Wu, Q. Meng, B. Xing, and S. Wang, PACIIA'08, IEEE, vol. 1, pp. 822-826,(2008).

2. M. Nixon, R. B. Havekost, L. O. Jundt, D. Stevenson, M. G. Ott, A. Webb, and M. Lucas, Process control system using a process control strategy distributed among multiple control elements, Feb. 27 (2001).

3. M. Nixon, L. O. Jundt, R. B. Havekost, and R. Ottenbacher, Process control system for monitoring and displaying diagnostic information of multiple distributed devices, Nov. 30 (1999).

4. Gajanan Kolmare, Prasad Bhase, Amruta Deshpande, International Conference on Computing, Communication and Automation (ICCCA), pp. 977-981, (2016).

5. Li Xin, Wang WenBing, Xu Jun, Proceedings of the 35th Chinese Control Conference July 27-29, (2016).

6. Jin LIU, Pei-Jian ZHANG ,Jian-Guo WU, Ping-fan BU, Wei-feng CHEN, Chinese Control and Decision Conference, IEEE Proceedings, (2016)

7. Luo YuXiong, Zhao Huimin, Shen Zhongli, Zhou Yucai, International Conference on Intelligent Computation Technology and Automation, IEEE Computer Society,(2008).

8. Hemangi Laxman Gawand, N. Murali and P. Swaminathan, International Conference on Reliability, Safety \& Hazard (ICRESH), (2010).

9. Ivan Castillo and Thomas F. Edgar, American Control Conference, (2009).

10. Hui Chang - Kun, Proceedings of the IEEE International Conference on Industrial Technology, (1996).

11. Edwin Pruna, Santiago D. Mullo, Jhonathan A. Caicedo, CHILEAN Conference on Electrical, Electronics Engineering, Information and Communication Technologies (CHILECON),IEEE Proceedings,(2017).

12. Khin Nway Oo, Zaw Min Naing, Hla Myo Tun, International Journal of Scientific \& Technology Research, vol.3, Issue 6, June (2014) 